\title{
Los desafíos de la educación médica en México
}

\section{The challenges of medical education in Mexico}

\author{
José Eduardo Erasmo García Luna Martínez', Luis Alberto Silva Ulibarri', Jorge Luis García Macías², Jesús Daniel López Tapia², \\ Ana Yolanda Sandoval Mussi², Olinda Giselle Garza Sáenz²
}

\section{Resumen}

La educación médica genera impacto en la salud de las poblaciones; formar médicos profesionalizados con capacidad para adaptarse a entornos cambiantes permite comprender con mayor detalle la epidemiología de una comunidad o país y mejorar la salud pública. Dar al estudiante de medicina las herramientas necesarias para proporcionar una atención de calidad puede mejorar la salud de cientos de pacientes. La educación médica está llena de desafíos y se encuentra en una búsqueda continua de excelencia. El modelo educativo actual busca centrarse en las competencias requeridas por los sistemas de salud y educación de México, pero COVID-19 rompió paradigmas y revolucionó la manera en la que se enseña medicina, centrándose en el aprendizaje autorregulado. El mundo está en constante cambio y la educación médica debe evolucionar con el mundo. La tecnología es la herramienta que revoluciona la enseñanza y permite introducir la globalización a las escuelas, aprendiendo así sobre otras culturas y su manera de practicar la medicina. La salud mental de los estudiantes es determinante para evitar la deserción y formar médicos sanos. El integrar condiciones de bienestar a nivel nacional e institucional tendrá un impacto positivo en la vida de los estudiantes, y los ayudará a desempeñarse mejor en el futuro. El reto de la educación médica actualmente es inspirar la formación de médicos capaces de insertarse en un mercado laboral competitivo, sin descuidar la atención al paciente, con habilidades directivas y organizacionales, evitando la sobre-especialización como limitante del desarrollo profesional.

Palabras clave: educación; salud; medicina; desafíos; tecnología; México.

\begin{abstract}
Medical education creates an impact on the health of the population. To train professional doctors capable of adapting to changing environments allows a more detailed understanding of the epidemiology in a community or country; and improves public health When medical students have the tools needed to provide quality care, it can improve the health of hundreds of patients. Medical education is full of challenges and is in a continuous search of excellence. The current educational model focuses on the skills required by Mexico's health and educational systems, but COVID-19 broke paradigms and revolutionised the way we teach medicine, focusing on self-regulated learning. The world is in constant change, and medical education has to evolve with the world. Technology is a tool that revolutionises teaching and allows us to introduce globalisation to schools while learning about other cultures and how they practice medicine. Focusing on the mental health of students is a determining factor in training healthy doctors and avoiding desertion. Incorporating well-being conditions at hospitals and schools can have a positive impact on the lives of the students, helping them have a better performance. The challenge of medical education today is to inspire the training of doctors capable of inserting themselves in a competitive labour market without neglecting patient care and with managerial and organisational skills, avoiding over-specialization as a limitation of professional development.
\end{abstract}

Keywords: education; health; medicine; challenges; technology; Mexico.

Fecha de envío: 10-26-2021 - Fecha de aceptación: 2021-12-14

(1) Vicerrectoría de Ciencias de la Salud, Universidad de Monterrey. México

(2) Escuela de Medicina, Universidad de Monterrey. México

Autor de correspondencia:eduardo.garcialuna@udem.edu 


\section{Introducción}

"La educación superior es un bien de carácter estratégico para las naciones, en tanto es el vínculo indisoluble entre la generación de capital humano altamente capacitado y la producción y difusión de conocimientos que favorecen la conformación de sociedades más justas y economías más competitivas" (Fernández, 2017).

Determinar la dirección y visualizar los objetivos de la educación médica hoy en día son temas que se presentan constantemente. No obstante, definir el futuro de los próximos médicos no es tarea sencilla debido al dinamismo del mundo actual y los constantes cambios sociales y culturales de cada país. Según Densen (2011), para avanzar en la misión de la educación médica es necesario contar con un liderazgo institucional decisivo y capaz de abordar los desafíos tanto internos como externos con una visión clara para la tercera década del siglo. Tomar los modelos educativos de diferentes países primermundistas e intentar aplicarlos a nuestra sociedad presenta una muralla de incógnitas a causa de no encontrarnos ante las mismas variables de cambio.

Apodaca-Orozco et al. (2017) tomaron en consideración uno de los principales retos actuales en la educación médica: alcanzar la excelencia, calidad y pertinencia, a través de un nuevo modelo educativo que toma en cuenta las competencias científicas, tecnológicas y humanistas de los estudiantes. Con esto los alumnos forman un compromiso social donde pueden adquirir conocimientos de manera integral, con esto se pueden desenvolver como profesionales competentes y con preparación científica para aceptar los retos de la sociedad moderna. Al tener un amplio desarrollo humanista y de ética, se puede instruir valores que prevalecen en las carreras de los futuros médicos (Cabrera, 2008).

Tener la oportunidad de intervenir en el futuro teniendo acción en el presente, representa una satisfacción interna para todo aquel que se dedica a la educación; en la educación médica, dejas de tener influencia sobre la salud de un paciente, y empiezas a tener impacto en la salud de cientos de pacientes al dar al estudiante médico las herramientas adecuadas para otorgar una atención médica de calidad. El objetivo de este artículo es presentar una visión general de los desafíos que viven los médicos en México durante su formación académica y el ejercicio de su profesión.

\section{Discusión}

\section{La educación médica en el México moderno}

En la década de los años 80, Ramsbottom-Lucier et al. (1999) consideraban que, en ese momento, las metas en la educación médica deberían enfocarse en romper el paradigma de la especialización médica y la comunicación entre los diferentes niveles de atención, con el fin de disminuir el tiempo de atención del paciente usando las recién aplicadas ciencias informáticas. Además, agregar al currículo universitario materias que ofrecieran habilidades administrativas y de marketing para sus estudiantes médicos, sin embargo, a pesar de los años pasados no se ha logrado este objetivo.

De manera puntual, Frenk et al. (2010) dividió las reformas educativas en medicina de los últimos 100 años en tres grandes períodos basándose en los modelos institucionales e instruccionales. El periodo inicial abarca desde el inicio del siglo pasado hasta los años 60 que toma como base la educación científica, buscando mantener una instrucción centrada en ciencia y una institución con bases en el centro educativo o universidad. Posteriormente, con la aparición del modelo de aprendizaje fundado desde la solución de problemas, traslocando la institución al aprendizaje vivencial con pacientes reales y simulados en ambientes hospitalarios.

A inicios del siglo XXI hasta la actualidad, el modelo educativo gira en torno a las competencias con una institución basada en los sistemas de salud y educación, teniendo como punto central el desarrollo de habilidades informáticas, salud pública, liderazgo, visión centrada al paciente y no a la enfermedad, y el trabajo interdisciplinario.

Gordon (2014) enumeraba varias preguntas que debíamos realizarnos en ese momento para determinar las directrices de la educación médica. Los cinco desafíos que él mencionaba eran ¿Qué función cumple un médico? ¿cuántos médicos necesitamos? ¿Cuántos médicos están siendo educados en este momento y qué tan bien están siendo educados? ¿cómo educamos médicos a estándares globales aceptables cubriendo las necesidades de su localidad? y ¿cómo nos aseguramos que los médicos tengan una visión holística de la salud?

Por otro lado, los profesores de las escuelas de medicina son un pilar en la formación de los alumnos. Campos (2016) menciona que ellos son un elemento clave para una educación médica de excelencia. Lo ideal es que los profesores posean características claves para apoyar a los alumnos en su formación. Deben ser creativos, capaces, resolutivos, y que posean disposición, que se preocupen y sobre todo que se dediquen a sus alumnos con vocación intelectual que despierte el sentido de reflexión e interés en los estudiantes. Es importante recalcar que los profesores deben ser facilitadores de la información. Uno de los desafíos más importantes en la enseñanza médica es el aprendizaje autorregulado y autodirigido. La enseñanza centrada en el estudiante reconoce a los estudiantes como protagonistas del proceso de aprendizaje; el profesor da las herramientas clave a los alumnos y ellos las utilizan para poder adaptarse a las circunstancias y poder optimizar la manera en la que aprenden, teniendo una mayor responsabilidad en este proceso (Abarca \& Bolton, 2007). 
Actualmente en el año 2021, varias de estas preguntas siguen sin respuesta y hasta se han vuelto obsoletas debido a los retos que enfrentamos de manera súbita debido a la pandemia por COVID-19. Con el estado epidemiológico debido al virus, nos vimos en la necesidad de dar un salto en la tecnología y transformar la forma en la que vemos la educación médica. Esto nos ha llevado a explorar técnicas educativas que esperábamos adoptar a futuro. Las preguntas antes planteadas, se deben de responder, pero en un entorno digital y totalmente nuevo. Tomando esta experiencia como una oportunidad, hemos tenido de primera mano experiencias que nos dan información en bruto que debemos analizar y dirigir hacia un punto en concreto, logrando una piedra angular en cómo educamos a los futuros médicos en la actualidad.

\section{EI COVID-19 y la educación médica}

La "generación COVID-19" de estudiantes médicos se presenta ante un reto importante en su educación condicionado por el aislamiento social y el desprendimiento de la práctica médica, así como la potencial despersonalización. Rose (2020) cuestiona ¿qué podrían hacer los educadores para crear experiencias para los estudiantes que son asignados a rotaciones clínicas?. Debido a la pandemia, los profesores se encuentran ante la gran tarea de encontrar las herramientas para desarrollar las competencias clínicas indispensables de sus alumnos.

El inicio de la pandemia actual marcó un precedente, según Aldo Barajas-Ochoa et al. (2020), el sistema de educación médica en México se debería adaptar a los requerimientos de salud ocasionados por la pandemia desde el internado de pregrado, el servicio social y las residencias médicas. Múltiples sistemas de salud tuvieron que adecuarse para disminuir la transmisión intrahospitalaria por medio de modificaciones tales como cancelar procedimientos quirúrgicos electivos, transformar quirófanos en unidades de cuidados intensivos, entre otras; viendo una disminución en el desarrollo de capacidades de los médicos en formación debido a la suspensión de las rotaciones clínicas, la transgresión directa a la esencia misma de la práctica médica, la relación médico paciente y el aprendizaje vivencial.

Derivado de esta situación, la gran mayoría de las escuelas de medicina optaron por el aprendizaje en línea a distancia. Gill (2020) menciona que esta forma de aprendizaje ya había sido previamente evaluada y aprobada para permitir una flexibilidad de horarios, educación internacional y un costo-beneficio mayor. Al transformar el aprendizaje a una modalidad totalmente en línea, nos encontramos ante estudiantes que no tuvieron prácticas anatómicas cadavéricas, estudiantes que carecen de habilidades manuales al no ser sometidos a prácticas médicas, y de experiencias vivenciales para el desarrollo de habilidades interpersonales con otros médicos y con pacientes.
Pero, no todo es negativo con respecto a esta situación epidemiológica actual y la educación médica. Gracias a esta situación pudimos romper paradigmas en la comunicación entre profesores y estudiantes, se ha logrado hacer una transición importante del modelo educativo clásico a un modelo digital con el uso de herramientas tecnológicas, experiencias en línea, administración del tiempo y disminución de la tecnofobia en los profesores. Además, Vergara et al. (2020) mencionan la importancia de la autoformación y disciplina que los propios estudiantes han tenido que asumir, practicando el aprendizaje autodirigido y mejorando así la forma en la que estudian y aprenden.

Nos encontramos en un momento que nos permite experimentar en temas de educación en línea, uso de realidad virtual, educación a demanda, experiencias internacionales desde la comodidad de la casa o simulación clínica en un ambiente sin riesgo y estandarizado. Con la participación de los estudiantes en los centros de simulación clínica, se adaptan las necesidades de entrenamiento de los alumnos y facilitan la evaluación de los conocimientos clínicos sin poner en riesgo a los estudiantes (Maestre et al., 2021).

Los educadores deben terminar con la tecnofobia como paradigma a romper, queramos o no la era digital es la nueva normalidad. La globalización de la salud y el acceso a educación internacional nos da la oportunidad de experimentar de primera mano cómo se hacen las cosas en otros países, dándonos la posibilidad de contestar varias de las preguntas angulares en la educación médica: ¿estamos preparando médicos globalmente competentes? ¿cuántos médicos necesitamos? ¿están bien educados? ¿tienen nuestros estudiantes una visión biopsicosocial de los pacientes y su enfermedad?

\section{La medicina digital}

La cuarta revolución, caracterizada por la combinación e integración de las tecnologías, ha ofrecido importantes avances a la medicina, sin embargo, estos avances han generado nuevos retos para la educación médica, la ética profesional y las políticas públicas.

Esta revolución o también llamada industria 4,0, aplicada al sector de salud, busca mejorar el acceso, la oportunidad y la calidad de los servicios de salud. Hasta la fecha, estos avances tecnológicos como la telemedicina y la bioimpresión 3D de órganos y tejidos han permitido brindar nuevas formas de atención a los pacientes, con sus consecuentes retos.

Hincapié \& Amariles (2019) identifican tres tendencias relevantes en el sector salud relacionadas con la industria 4,0. La primera tendencia es la inteligencia artificial; implementada mediante modelos de aprendizaje automatizado donde estos sistemas desarrollados tienen la capacidad de diagnosticar una enfermedad de manera más precisa por medio de 
máquinas que detectan datos en imágenes o software que apoyan al médico tratante a intervenir de la manera más adecuada. La segunda tendencia identificada es la medicina de precisión o personalizada; un conjunto de tecnologías que utilizan la información de la secuenciación del genoma humano para identificar o tratar las enfermedades. Se espera que en la próxima década los productos bioterapéuticos estén comercializados a gran medida, así como las terapias dirigidas, celulares y génicas. Por último, tenemos la tendencia de la big data e internet de las cosas; el empleo del expediente clínico digital con la recolección de información de los pacientes nos obliga a una evolución de las políticas públicas en materia de salud.

En Colombia se ha implementado una "carpeta ciudadana" que funciona como un espacio en la nube donde se almacena información clínica de cada ciudadano. La big data se relaciona principalmente con el desarrollo de robots o dispositivos vestibles. Un gran ejemplo de los dispositivos vestibles son los que registran los niveles de glucosa sérica y almacenan la información en un dispositivo o nube donde pueden ser consultados por el médico tratante o el mismo paciente. Esta herramienta permite que el médico tome decisiones sin necesidad de una consulta presencial, además de facilitar la predicción de riesgos e identificación del perfil de cada paciente. Los retos que principalmente se identifican debido a la era 4,0 son los altos costos para financiar los sistemas de salud con algunas de estas tecnologías, así como la privacidad, seguridad, gobernanza y análisis de datos que tendrán que ser discutidos para su utilidad.

\section{Aprendizaje médico global}

La internacionalización es un proceso que se ha orientado a introducir la multiculturalidad, con la finalidad de mejorar las metas, funciones y entrega de la educación superior, e impulsar así la calidad académica y la investigación (Fenoll-Brunet, 2016). El incremento de facultades de medicina a nivel mundial, la diversidad poblacional, la internacionalización del mercado laboral médico y el impacto de la globalización en el ámbito de la atención sanitaria nos ayudan a corroborar todas las necesidades de implementar estándares globales para la formación médica y evaluar la adquisición de competencias adecuadas para ejercer en cualquier lugar del mundo. El conocer otras culturas y aprender de ellas, ayudan a mejorar la calidad de trato a los pacientes y a que los estudiantes aprendan diferentes formas de hacer medicina, que les permitan generar soluciones ágiles frente a escenarios cambiantes.

El tener una comunicación directa con escuelas de medicina a través del mundo, ayuda a que los estudiantes puedan aprovechar oportunidades de estudio y aprendizaje conocimiento que en otro tiempo no hubieran adquirido. Las herramientas que las escuelas de medicina obtengan son clave para que se revolucione la educación de sus estudiantes.
Es indispensable que se adopte una perspectiva internacional a partir de estándares de calidad en educación médica de pregrado, posgrado y educación continua, que incluyan indicadores que permitan valorar los niveles alcanzados de internacionalización. De igual forma, se requiere adoptar estándares de calidad universalmente aceptados para evaluar la educación médica de pregrado a nivel internacional donde se promueva la realización de cambios para poder compartir recursos y experiencias. Esto supone un nuevo reto frente a las políticas de salud de cada país y la necesidad de romper fronteras en temas de salud pública y educación médica.

\section{Salud mental en los estudiantes de medicina}

En relación a los desafíos que puede presentar estudiar medicina, Steiner-Hofbauer \& Holzinger (2020) comentan que del 20,9\% al $90 \%$ de los estudiantes de medicina experimentan estrés. Los factores causales más comunes son la alta carga de trabajo, la amplitud del programa académico, la frecuencia de los exámenes, el aislamiento social, poco tiempo libre, las altas expectativas de sí mismos y la competencia con sus compañeros. Por consiguiente, el consumo de sustancias se ha visto aumentado en esta población y esta prevalencia no se ve disminuida al ingresar a la vida profesional como médicos. Slavin \& Chibnall (2016) mencionan la importancia de restaurar los entornos educativos y clínicos con la finalidad de reducir los factores estresantes innecesarios, creando un ambiente académico más positivo tanto para el aprendizaje como para la atención médica.

Los autores Slavin \& Chibnall (2016), afirman que la educación médica de posgrado continúa siendo una etapa donde se observa el estrés, la depresión, la ansiedad y las ideas de autoagresión en los médicos residentes de todas las especialidades. Asimismo, se cuestiona la posibilidad de apoyar tanto a los estudiantes de medicina como a los médicos residentes a encontrar su significado en el trabajo, para de esta manera mejorar su salud mental o por lo menos detener su deterioro. Slavin \& Chibnall (2016) ejemplifican la vida de Viktor Frankl un psiquiatra, neurólogo y autor que pasó 3 años en cuatro campos de concentración durante la Segunda Guerra Mundial, argumentó que no existe nada más efectivo que ayude a sobrevivir incluso ante las situaciones más difíciles que el conocimiento del significado de su vida. Con base en este pensamiento se desprende una pregunta importante: ¿podemos diseñar intervenciones educativas para sostener a los residentes a encontrar y sentir su por qué?

En la experiencia de los autores, existen 3 principales razones que pueden limitar el aprendizaje de los estudiantes de medicina: primero, cambiar constantemente el entorno de aprendizaje. La diversidad de ambientes académicos resultante de las continuas rotaciones en escenarios clínicos, expone a los estudiantes de 
pregrado y posgrado a tutores con distintos niveles de formación y estrés, en algunos casos sin una evaluación psicológica apropiada, y esto puede reflejarse en sus prácticas docentes con estrategias que afecten el aprendizaje, así como generar o exacerbar trastornos de salud mental en los estudiantes. Segundo, las estrategias posibles para reducir el estrés se vuelven más complicadas de utilizar durante los últimos años de la escuela de medicina (internado de pregrado o pasantía). La capacidad para auto-reconocer problemas de salud mental va disminuyendo al mismo tiempo que se va volviendo más sólido el perfil profesional. Finalmente, el aumento en la competitividad y la presión de ingresar a una residencia médica durante el último año de la escuela de medicina va separando o incluso rompiendo lazos de intercomunicación con los compañeros, incrementando el nivel de estrés, ansiedad e incluso la percepción de soledad.

Slavin \& Chibnall (2016) establecen que será necesario cambiar las condiciones de bienestar a nivel nacional e institucional, por medio de intervenciones guiadas por las experiencias de los propios alumnos y residentes, así como analizar estudios que evalúen el impacto de las intervenciones para de esta manera ayudar a los médicos en formación a manejar el estrés y encontrar significado en su trabajo. Además, será necesario que ciertas intervenciones se basen en reducir el ambiente tóxico en las instituciones educativas y el campo clínico, "con datos y compromiso con el cambio, podemos comenzar a avanzar para crear un nuevo paradigma en la educación médica y en la atención de la salud que apoye en lugar de disminuiry que inspire en lugar de desanimar" (Slavin \& Chibnall, 2016).

\section{La inserción al mercado laboral y a la educación del posgrado}

La Secretaria de Salud en México encontró que un desafío de gran importancia para la educación médica en el país, es la formación de médicos orientados hacia una atención primaria y menos enfocada en la sobre-especialización de su profesión (Molina et al., 2021). Sin embargo, para que esto sea posible, primero ve necesario modernizar e integrar en una sola estructura organizacional el sistema nacional de salud. Lo anterior, a través de un modelo estandarizado de atención primaria de la salud que garantice un servicio de calidad homogéneo entre las distintas instituciones del sector. El éxito de este modelo dependerá en gran medida de la continuidad que le brinde la administración entrante durante cada cambio de gobierno.

Por otro lado, es prioritario que durante la modernización del sistema de salud se amplíe el financiamiento necesario para la infraestructura hospitalaria y la contratación de capital humano suficiente para crear equipos de salud multidisciplinarios reforzando así la atención sanitaria de primer nivel. Es necesario también la creación de redes interinstitucionales para coordinar sus responsabilidades y romper paradigmas de salud pública y ciertos determinantes sociales que solo competen al sector salud. La promoción de la salud y la educación médica deben reforzarse, para que la población general conozca cómo cuidar su salud desde casa y haga sostenible la atención primaria en las diferentes unidades clínicas.

Esta transformación será posible si inspiramos la formación de gestores sanitarios que tengan habilidades directivas, organizacionales y de salud pública con el propósito que el gobierno institucional tenga los conocimientos necesarios para impulsar el desarrollo de este vanguardista sistema nacional de salud. Una estrategia concreta para ejecutar esta transformación podría ser, tomar la experiencia adquirida en el programa IMSS Bienestar realizando un análisis en la búsqueda de las fortalezas, oportunidades, debilidades y amenazas. Con el propósito de focalizar los esfuerzos en aquello que hace valioso el programa y adaptarlo a este un nuevo modelo de salud pública que trabaje en conjunto con las instituciones formadoras de capital humano en salud; para que esta a su vez éstas alineen sus programas académicos de manera que éstos respondan a las necesidades actuales de la población en materia de atención médica primaria.

\section{Conclusión}

Los desafíos para la educación médica en México, moderno o mejor dicho "post-COVID", no serán una tarea fácil. pero tampoco una novedad absoluta que nos haya confrontado de manera súbita. La introducción de nuevas tecnologías será determinante, como el uso de simulación, que ha sido largamente experimentado en el desarrollo de competencias profesionales dentro del proceso educativo de nuevos profesionales de la salud a nivel de pregrado, especialidades médicas, incluso profesiones afines. Si bien las experiencias han sido compartidas a través de eventos académicos como congresos o simposios, la educación basada en el uso de nuevas tecnologías no ha substituido la práctica simulada ni la experiencia clínica en escenarios reales. Este reto implica una amplia diversidad de recursos disponibles en las instituciones de educación médica.

Otro formato relevante a considerar es la educación a distancia o en línea, la cual ya es considerada bajo ciertos criterios de entidades acreditadoras como la Red Iberoamericana para el Aseguramiento de la Calidad en la Educación Superior (RIACES), marcando con ello una nueva etapa en la conceptualización de criterios de calidad en la educación médica y sus procesos. Más aún, se debe reconocer la agilidad en los procesos de cambio que el entorno impone al quehacer formativo. 
No debe desdeñar la importancia en los balances de vida de los procesos de formación y ejercicio profesional que la experiencia de la pandemia y las modalidades a distancia y en línea han generado; ya sea el aislamiento, la potencial despersonalización o el desapego al ejercicio de la medicina deberán considerarse como elementos relevantes de calidad del proceso educativo.

También vale la pena preguntarse sobre el rol que la inteligencia artificial jugará en el quehacer profesional futuro y, en un futuro más cercano, en los procesos formativos de nuevos médicos. Ello nos lleva a la pregunta sobre cuáles serán las competencias digitales requeridas para ello. El entorno laboral para el médico recién egresado, ya formado o en proceso de especialización a través de residencias médicas se verá igualmente trastocado por lo antes dicho, evolucionando hacia formatos similares.

Finalmente, existe un cambio implícito de paradigma en la manera cómo interactúan formadores y formados, migrando de una estructura de interacción vertical, basada en la jerarquía, hacia otra horizontal centrada en el acompañamiento y consejería.

\section{Referencias}

Apodaca-Orozco Ginne Ussi G, Ortega-Pipper LP, VerdugoBlanco LE. \& Reyes-Barribas LE. (2017). Modelos educativos: un reto para la educación en salud. Ra Ximahai 13, 1-11.

Barajas-Ochoa A, Andrade-Romo J. \& Ramos-Santillán V. (2020). Challenges for medical education in Mexico in the time of COVID-19. Gaceta médica de México 4.

Cabrera AP. (2008). Ética y humanismo en la formación médica. Acta Bioethica, 14, 30-38.

Campos A. (2016). Las cuatro ruedas del carro de la excelencia. Desafíos y limitaciones en la educación médica. Educación Medica 17, 1-6.

Densen P. (2011). Challenges and opportunities facing medical education. Transactions of the American Clinical and Climatological Association 122, 48-58.

Dolci GF. (2019). Editorial: La educación interprofesional, la simulación clínica y la seguridad del paciente. Suplemento Revista de La Facultad de Medicina 62, 5-7.

Fenoll-Brunet MR. (2016). El concepto de la internacionalización en la enseñanza superior universitaria y sus marcos de referencia en educación médica. Educación Médica 17, 1-9.

Fernández E. (2017). Una mirada a los desafíos de la educación superior en México. Innovación Educativa 17, 183-207.
Frenk J, Chen L, Bhutta ZA, Cohen J, Crisp N, Evans T, Fineberg H, Garcia P, KeY, Kelley P, Kistnasamy B, Meleis A, Naylor D, Pablos-Mendez A, Reddy S, Scrimshaw S, Sepulveda J, Serwadda D. \& Zurayk H. (2010). Health professionals for a new century: transforming education to strengthen health systems in an interdependent world. Lancet 376, 1923-58.

Gill D, Whitehead C. \& Wondimagegn, D. (2020). Challenges to medical education at a time of physical distancing. Lancet 396, 77-79.

Gordon David. (2014). What is medical education for? The challenges in global medical education today. EMHJ-Eastern Mediterranean Health Journal 20, 149-150.

Hincapié García JA. \& Amariles Muñoz-, P. (2019). Cuarta revolución industrial:Tendencias en salud. Revista digital: Universidad de Antioquia. Accedido en : https://www.udea.edu.co/wps/portal/udea/web/ inicio/udea-noticias/udea-noticia/!ut/p/z0/fY8xC8IwEIX_ikvHklhr1LE4COLgINJmkSMJeprm2jQp_nxTHcTF5XHv-N6DxySrmXQw4hUCkgObfCPFZb3ZFvOq5AcuSsErcSyXq2K3OJ052zP5H0gNeO97WTGpyAXzDKzuyAewURvIOAy_7kat-dyTzhwFVAhDxt9ph5om6vvujEfSqAhsCy0E4xMaTarMvRnJRpWW5Oh0HILHNKI7yOYF7YV8iA!!/ el 13 de septiembre de 2021.

Maestre JM, Rábago JL, Cimadevilla B, Pedraja J. Moral ID. \& Manuel-Palazuelos JC. (2021). La simulación como herramienta para facilitar la adaptación de la organización sanitaria a la pandemia de COVID-19. Educación Médica 22.

Molina Leza J, Ruiz Larios JA, Zamora Ramos E. \& Bejarano Medellín R. (2021). Desafíos de la Atención Primaria de Salud en México. Coordinación de Análisis Estratégico y Evaluación. Gobierno de México, pp 2-45. Accedido en : https://www.gob.mx/cms/uploads/attachment/file/651873/Informe_Desaf_os_APS_ENERO_2021_final.pdf el 10 de septiembre de 2021.

Ramsbottom-Lucier M, Pregler J. \& Gomez AG. (1999). Challenges in medical education: training physicians to work collaboratively. Journal of general internal medicine 14, S21-S25.

Reyes Abarca, C., \& Moreno Bolton, R. (2007). Enseñanza centrada en el estudiante. ARS MEDICA Revista de Ciencias Médicas 7, 107-119.

Rose S. (2020). Medical Student Education in the Time of COVID-19. Jama 323, 2131-2132.

Slavin SJ. \& Chibnall JT. (2016). Finding the Why, Changing the How. Academic Medicine 91, 1194-1196. 
García et al.

Steiner-Hofbauer V. \& Holzinger, A. (2020). How to Cope with the Challenges of Medical Education? Stress, Depression, and Coping in Undergraduate Medical Students. Academic Psychiatry 44, 380-387.
Vergara E, Vergara, R, Alvarez M, Camacho L. \& Galvez, J. (2020). Educación médica a distancia en tiempos de COVID-19. Educación médica Superior 34. 